\title{
FiY1: A reflective account of the foundation interim year 1 post
}

\author{
Authors: Imogen Jones ${ }^{A}$ and Gregory Neal-Smith ${ }^{B}$
}

\begin{abstract}
During the COVID-19 pandemic, many final-year medical students were qualified early with the voluntary option to join the workforce as foundation interim year 1 (FiY1) doctors. In this column, a foundation year 1 (FY1) doctor reflects on their FiY1 experience of starting work as a practising doctor in the height of a pandemic. The process of the induction, the structure of the job, and the overall experience of an FiY1 doctor is reviewed and reflected on against previous years. The author aims to shed light on the FiY1 role for students who may find themselves in similar positions in the future.
\end{abstract}

KEYWORDS: coronavirus, pandemic, foundation year 1, interim, students

DOI: 10.7861/clinmed.2020-0921

\section{Introduction}

The first Wednesday in August is colloquially known as "Black Wednesday'. It marks the annual admission of freshly qualified junior doctors at their allocated hospitals, all beginning their first day as a foundation year 1 (FY1) doctor. However, the first Wednesday in August 2020 was somewhat different from previous years, as many final year medical students had graduated early with the opportunity to take an 'interim' post during the COVID-19 pandemic. This meant that many of the new cohort of junior doctors had been practising medicine for up to 8 weeks prior to starting FY1. These posts were given the name foundation interim year 1 (FiY1). This column is written by a doctor who undertook an FiY1 post at a small district general hospital and their unique experience of starting work during a pandemic is reflected upon. The author aims to offer an insight into the FiY1 role for current medical students who may find themselves in a similar situation in the future.

For this novel job role, the UK Foundation Programme set out employment arrangements for trusts accepting FiY1 doctors. Final year medical students were offered the chance to apply to a deanery to work as an FiY1. There was no exam or competitive

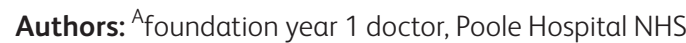
Foundation Trust, Poole, UK; ${ }^{B}$ core surgical trainee (CT2), Royal Berkshire Hospital, Reading, UK application process, but deaneries would accept new doctors on a first come, first served basis. The broad guidelines of the FiY1 employment arrangements stated that FiY1s should have an induction, be well supervised, receive remuneration, have additional support, be assigned a named clinical supervisor and have access to the foundation training eportfolio. ${ }^{2}$ Unlike with FY1s, there was no requirement to undertake clinical assessments as there was no educational sign off at the end of the placement.

\section{Induction}

The FiY1 induction was a fast-track of the usual FY1 induction, condensed into one day as opposed to one week (the usual length of FY1 induction at the same hospital). This equipped us with a very basic knowledge of the local trust policy, logins and rota schedules and the fundamentals of life support. We were introduced to the chief registrar, who was to be a port of call for escalating issues from juniors to senior staff. We were taught how to put out a crash call if there was an emergency; however, we were not formally told how to bleep other doctors or who to escalate daily clinical queries to (presumably due to the fact that joining doctors would be based on different specialties and escalation of clinical issues would be taught on the individual wards). The induction did not include protected time shadowing FY1s, lectures on medical emergencies, practical skill sessions or familiarisation with the wards. These are areas typically covered in the traditional weeklong FY1 inductions, which can help reassure many of the joining doctors. A memorable part of the FY1 induction that some trusts have run in previous years is the simulated 'on-call bleep holder' sessions, which provide an opportunity for those undergoing full induction to practice prioritising and responding to bleeps. For those on the FiY1 induction this opportunity was not available, and the first time they carried a bleep was on their second day. From a social aspect, having only one day for the FiY1 induction meant that the new doctors began shifts without knowing many of their fellow colleagues. This reduced the opportunity to form the colleague support networks that may be gained from longer induction sessions, but most of the FiY1s were in the same position and joining teams were aware of the situation, which eased the transition into work.

After the FiY1 induction, I found that the most helpful advice was gained 'on the job' from the doctors already working on the wards. This included guidance on prescribing medications and completing discharge summaries, and tailored advice on how the consultants run their ward rounds. This is probably not 
too different from the usual FY1 experience, as it is impossible to prepare new doctors with everything they need to know before starting work. However, I feel the induction could have involved more time shadowing the FY1s on the ward and being supernumerary prior to starting work, although as we were beginning work in the first wave of a pandemic where staffing was already short, this might not have been a realistic expectation. To supplement shortened inductions in future, there are online resources that can aid FiY1 doctors starting in new trusts, with useful suggestions to ease the transition into working. ${ }^{3}$

\section{The job}

The first day as a practising doctor ( $\mathrm{FiY} 1$ or FY1) is an immediate step up in responsibility from medical school. On my second day as an FiY1 I remember being approached by a nurse who asked, 'Doctor, can you...' and I looked over my shoulder thinking they must have been speaking to someone else. The FiY1 post I undertook did not include night shifts, on-calls or weekend shifts. This had the benefit of ensuring some personal time was protected and helped prevent work-related burnout. There were, however, still significant expectations in the FiY1 role, as some of us found ourselves in difficult situations caring for patients with little available support. Often, I found myself to be the only doctor accompanying the consultant on the ward round. I would be expected to document precisely to their individual preferences, complete patient reviews and jobs. This may be comparable to some FY1 experiences, although the COVID-19 pandemic put an obvious strain on clinical staffing and meant that support levels of middle grade or other doctors were sparser in some departments than usual. Although FY1 and particularly FiY1s should not be left unsupported in their role, in practice it has happened on occasion. This made it more difficult at times to escalate clinical problems, as often seniors were not around to review patients. I found the best strategy was to fall back onto the Resuscitation Council's A to E approach for assessing acutely unwell patients. ${ }^{4}$ I found that no one ever criticised this, so if you are in doubt, I would recommend having a safe approach to fall back on such as this. If you assess the patient as stable, it reassures you and the nurses, and any further management is often safe to wait until your senior becomes free.

\section{Overall experience}

At the time of writing, there is a second wave of COVID-19 occurring and an FiY1 placement is a possibility for another cohort of final year medical students. There are certain tasks and specific procedures which take time to learn at a new hospital: getting to grips with the computer systems, how to make referrals to different specialties and navigating around the hospital site. In fast-tracked inductions, it is difficult to fit all these tasks in as juniors are expected to begin work in a shorter time period. However, FiY1 (and later FY1) was an excellent opportunity for me to learn how to be a doctor and understand the responsibilities of the role. Furthermore, it meant working at a time where I felt I was making a real difference to the patients I saw and, in a wider view, to the hospital and the NHS. As a result, I found it an immensely satisfying and engaging experience. It is difficult to comment on how this experience might compare to an FiY1 starting in a tertiary centre, or in a similar district general hospital with different structures, but I believe there would be benefits to every FiY1 programme.
By doing FiY1, the theory I learned at medical school could be put into practice in a mostly supported environment. Despite all the pressures, there appeared to be good learning opportunities which prepared me well before starting FY1. I found this eased the transition from medical school to foundation training and, as a result, the start of FY1 seemed like an extension of the job that we had already been doing - especially as many of us were placed on the same ward for FiY1 that we had been due to start on first in FY1. This benefited the hospital in turn, as there were a significant number of FY1s already experienced in their starting rotation. Those that had worked in the trust for FiY1 were particularly useful to the new starters as sources of information and guidance on local policies. This meant that some socialising and support networks came from colleagues reaching out to each other for advice or assistance. With respect to patient care, the doctors who stayed on their wards were able to deliver nearly continuous patient care and the nursing and allied health professionals also benefited from the continuity of a professional relationship.

There may be a place for FiY1 in the future of the foundation programme beyond the pandemic, due to the significant benefits it offers in easing the transition from medical school and creating a cohort of more confident and competent junior doctors. How this would be addressed is a complex issue; it could involve representatives from hospitals and medical schools setting out clear expectations and goals that are achievable in the time frame. I would suggest appointing final year medical student representatives to act as a link between hospitals and their student cohort. That way, there is a clear passage of communication between the students and hospitals and a platform on which their views can be shared. With the resources available through large student national collaboratives, it could be beneficial to collect quantitative and qualitative data from former FiY1s before feeding back to improve the programme.

\section{Support}

Providing adequate support for FiY1s is a prerequisite set out by the UK Foundation Programme, as it is normal to feel apprehensive before starting a new job and experience challenges as the job begins, especially if it is brought on earlier than originally planned. FiY1s should have clinical supervisors appointed to them who can provide escalation and support. If this is not in place, it has been beneficial to some FiY1s to know who the British Medical Association junior doctor representative is for their hospital, as this individual can escalate clinical matters on behalf of junior doctors. ${ }^{5}$

Finding a life outside of work is imperative for good mental health - free versions of apps such as Headspace are helpful in winding down after a tough day. ${ }^{6}$ Talking to friends and colleagues can be a good way to deal with stress too; however, I recommend also being proactive and seeking out more formal support systems, eg clinical and educational supervisors, as they can guide you about how to respond to situations or clinical incidents. It is best practice to touch base with your supervisors as early as possible into your placement as they can be invaluable help in times of difficulty.

\section{Conclusion}

FiY1 is more than a novel job role for newly qualified doctors created in the wake of a pandemic. For me, it was a time to watch and learn about the job of a junior doctor, work out how to solve 
problems in a stretched system and take responsibility - all before starting FY1. FiY1 had challenging moments with varying support; however, the experience meant that as a new doctor I was better prepared to manage the pressures on the wards and how to respond to unfamiliar situations. During the FiY1 placement, I felt like I really made a difference to the patients I was caring for and, in the process, helped the department in a time when it was very overrun. Overall, the FiY1 placement meant I was able to confidently start my FY1 job and as a result I am grateful for the experience.

\section{References}

1 Gaskell N, Hinton R, Page R, Elvins R, Malin A. Putting an end to Black Wednesday: improving patient safety by achieving comprehensive trust induction and mandatory training by day 1. Clin Med 2016;16:124-8

2 United Kingdom Foundation Programme. Allocation to interim F1 posts in England. UKFP, 2020. www.pastest.com/media/2814/ukfoundation-programme-info.pdf [Accessed 9 December 2020]
3 Truelove A, Baldwin AJ. FiY101: A quick quide for newly qualified doctors. Clin Med 2020;20:442-4.

4 Resuscitation Council UK. The 2015 resuscitation guidelines: The $A B C D E$ approach. www.resus.org.uk/library/2015-resuscitationguidelines/abcde-approach [Accessed 9 December2020].

5 British Medical Association. BMA junior doctors committee UK overview. www.bma.org.uk/what-we-do/committees/junior-doctorscommittee/junior-doctors-committee-uk-overview [Accessed 3 September 2020].

6 Haidrani L. Headspace. Emerg Nurse 2017;24:15.

Address for correspondence: Dr Imogen Jones, Poole Hospital, Longfleet Road, Poole, Dorset BH15 2JB, UK. Email: imogenjones@doctors.org.uk 